\title{
CAPACIDADE COMBINATÓRIA DE NOVE LINHAGENS ENDOGÂMICAS DE MILHO (Zea mays L.) EM DOIS AMBIENTES
}

\author{
COMBINING ABILITY FOR NINE INBRED LINES OF MAIZE IN TWO \\ LOCATIONS
}

\author{
Ana Beatriz Locatelli ${ }^{1}$ Luiz Carlos Federizzi ${ }^{2}$ Valdemar Naspolini Filho ${ }^{3}$
}

RESUMO

\begin{abstract}
O milho é um cereal que faz parte de um grande número de produtos e apresenta produção crescente em nível mundial. A maior parte das áreas produtivas no Brasil são ocupadas por híbridos obtidos a partir de linhagens endogâmicas. Nos anos de 1991/92, foram conduzidos dois experimentos em blocos ao acaso e três repetições, com 36 híbridos de nove linhagens endogâmicas de milho em Não Me Toque e Tapera, ambos locais no Rio Grande do Sul, com o objetivo de avaliar estes híbridos quanto à capacidade combinatória em dois ambientes, para os caracteres altura de inserção da espiga superior, porcentagem de tombamento, prolificidade e rendimento de grãos. A significância da interação C.G.C. e C.E.C. com ambiente, evidenciou as diferenças de comportamento dos genótipos nos dois locais. As linhagens presentes nas melhores combinações foram as mesmas nos dois ambientes, mostrando que a C.G.C. foi um bom indicador do desempenho das linhagens em combinações híbridas, embora os melhores híbridos não tenham sido os mesmos nos dois locais. As linhagens que se destacaram foram ID01, IMD04, IMD06 e ID09.
\end{abstract}

Palavras-chave: rendimento de grãos, capacidade de combinação, interação genótipo x ambiente.

\section{SUMMARY}

Corn is present in a great number of products and its area and production is growing in the world. In 1991/92 two evaluation trials were conducted in a complete randomised block design and three replications, with 36 hybrids of nine inbred lines in "Não Me Toque" and "Tapera", both in the state of Rio Grande do Sul, with the aim of evaluating the genetic diversity and combining ability of these hybrids for ear height, stalk-root, lodging, prolificacy and grain yield. The significant interaction for CGA and SCA with location showed that the genotypes had

\begin{abstract}
different performance in the two environments. The inbred lines in the best combinations were the same at the two locations, showing that the CGA was a good indicator of inbred lines performance at hybrid combinations, although the best hybrids were not the same at the two locations. The best inbred lines were ID01, IMD04, IMD06 and ID09.
\end{abstract}

Key-words: grains yield, combining ability, genotype vs. environment interaction.

\section{INTRODUÇÃO}

Para a produção comercial de milho, é importante ter híbridos que possuam alto potencial de rendimento e que respondam favoravelmente quando cultivados em diferentes ambientes. As diferenças genéticas e ambientais contribuem para a variação fenotípica e para a interação dos genótipos com diferentes ambientes. A interação do genótipo com o ambiente reduz a correlação entre o fenótipo e o seu genótipo, restringindo a validade das inferências sobre o comportamento do ponto de vista do melhoramento e da herança de caracteres quantitativos. É importante avaliar as magnitudes das interações genótipos x locais e genótipos $\mathrm{x}$ anos, para orientar as estratégias de melhoramento e a recomendação de cultivares em função da estabilidade fenotípica dos genótipos para cada região.

\footnotetext{
${ }^{1}$ Engenheiro Agrônomo, Mestre, Bolsista de doutorado do CNPq, Faculdade de Agronomia da Universidade Federal do Rio Grande do Sul (UFRGS). E-mail: ablocatelli@annex.com.br.

${ }^{2}$ Engenheiro Agrônomo, PhD., Bolsista do CNPq, Professor do Departamento de Plantas de Lavoura, Faculdade de Agronomia da UFRGS, Av. Bento Gonçalves, 7712, CP 776, 90012-970, Porto Alegre, RS. E-mail: federizi@ufrgs.br. Autor para correspondência.

${ }^{3}$ Engenheiro Agrônomo, Mestre., Delta Pesquisa e Sementes. E-mail: naspolini@ seanet.com.br 
Em híbridos simples envolvendo linhagens previamente testadas, genes que condicionam a capacidade específica de combinação (CEC.) têm maior efeito em determinar diferenças de rendimento, sendo que em materiais não testados previamente, genes que afetam a capacidade geral de combinação (CGC.) têm maior importância (MUNGOMA \& POLLAK, 1988). Um milho híbrido ideal de duas linhagens seria aquele em que todos os seus locos fossem heterozigotos (VENCOVSKY, 1987).

A interação das capacidades de combinação com o ambiente tem maior importância quando se tratam de caracteres que têm baixa herdabilidade e que apresentam maior dificuldade em separar o que é devido à herança genética e o que é efeito de ambiente. A não significância da interação da CGC com o local permite a obtenção de estimativas adequadas dos efeitos aditivos dos genes para os caracteres de importância, em um só ambiente. A interação não significativa da CEC com o local demonstra a versatilidade do genótipo em obter um bom desempenho em diferentes ambientes. Já a significância desta interação indica que o genótipo tem adaptação específica a determinados ambientes de teste.

Trabalho de PANDEY et al. (1991) com dez populações tropicais de milho, mostrou que caracteres com alta herdabilidade como, florescimento, estatura de planta, altura de inserção da espiga, não requerem avaliação das progênies em muitos locais; mas a seleção para rendimento de grãos que apresenta baixa herdabilidade foi mais eficiente com testes em vários locais.

Já BECK et al. (1990) observaram interação significativa para genótipos $\mathrm{x}$ locais e CGC x locais, para os caracteres rendimento de grãos, dias até o florescimento feminino, estatura de planta e altura de inserção da espiga superior. A interação CEC $x$ locais foi significativa somente para estatura de planta. Estas informações permitiram a definição das melhores populações para servirem de base para o programa de melhoramento ou então serem cruzadas com genótipos dos locais em que foram avaliadas para posterior seleção.

Interação significativa dos genótipos com ambiente foi encontrada por NEVADO e CROSS (1990), para as características rendimento de grãos, número de espigas por planta, peso de 200 grãos e número de grãos por fileira, na avaliação de três dialélicos de oito sintéticos em nove ambientes por três anos, sendo que os efeitos aditivos foram significativamente mais importantes para essas características.

$\mathrm{Na}$ análise da capacidade combinatória de seis linhagens antigas e seis linhagens recentes,
MELCHINGER et al. (1990) observaram que a interação do ambiente com as CGC e CEC foi significativa somente para o grupo de linhagens mais antigas.

Os objetivos do presente trabalho foram testar as capacidades geral e específica de combinação entre nove linhagens endogâmicas de ciclo intermediário em dois ambientes e avaliar o potencial dessas linhagens e seus híbridos F1's.

\section{MATERIAL E MÉTODOS}

Foram utilizadas nove linhagens endogâmicas de milho de ciclo intermediário, provenientes do Programa de Melhoramento da Empresa Sementes Agroceres S.A.: ID01, IF02, IF03, IMD04, IMD05, IMD06, ID07, IF08, ID09, nas quais: $\mathrm{I}=$ ciclo intermediário, $\mathrm{D}=$ grão dentado, $\mathrm{MD}=$ grão meio-dente e $\mathrm{F}=$ grão duro. Foram realizados os 36 cruzamentos possíveis entre as linhagens.

Dois ensaios foram instalados em dois locais: Não Me Toque (RS), localizado a $28^{\circ} 5^{\text {, }}$ latitude sul e $53^{\circ} 7^{\prime}$ longitude oeste, à 460 metros de altitude, em área de coxilha e em Tapera (RS), situado a $28^{\circ} 6^{\prime}$ latitude sul e $53^{\circ} 2^{\prime}$ longitude oeste, à 310 metros de altitude em área tipicamente de várzea. Cada ensaio contou com 18 híbridos F1's e sete testemunhas comerciais, ambos com três repetições em blocos ao acaso. As testemunhas foram comuns aos dois ensaios. Cada parcela teve duas fileiras de 4 metros, espaçadas de 0,90 metros, com uma população de 55.500 plantas por hectare.

Foram avaliados os seguintes caracteres: altura de inserção da espiga superior $(\mathrm{cm})$, acamamento, prolificidade (número de espigas/planta) e rendimento de grãos (kg/ha, 13\%). As análises de variância dos ensaios foram realizadas conforme método apresentado por PIMENTEL GOMES (1990), que permite a análise conjunta de experimentos em blocos ao acaso com alguns tratamentos comuns. Na análise conjunta, a soma de quadrados para tratamentos foi decomposta em capacidade geral e específica de combinação, conforme Método 4, Modelo I proposto por GRIFFING (1956), que considera os tratamentos fixos. A interação da soma de quadrados de tratamentos com locais foi decomposta em interação da capacidade geral e específica com locais, conforme sugestão de VENCOVSKY \& BARRIGA (1992).

\section{RESULTADOS E DISCUSSÃO}

Foram avaliados os caracteres altura de inserção da espiga, prolificidade, porcentagem de tombamento e rendimento de grãos, considerados importantes características na obtenção de híbridos de milho. Na tabela 1, estão os quadrados médios 
Tabela 1 - Quadrados médios da análise de variância e dos componentes da capacidade combinatória (C.G.C. e C.E.C.), médias, coeficientes de variação, para os caracteres estudados nos F1's obtidos através de cruzamento dialélico de milho. Não Me Toque e Tapera/RS, 1992.

\begin{tabular}{|c|c|c|c|c|c|}
\hline \multicolumn{6}{|c|}{ QUADRADOS MÉDIOS } \\
\hline $\begin{array}{l}\text { CAUSAS DE } \\
\text { VARIAÇÃO }\end{array}$ & GL & $\begin{array}{l}\text { ALTURA INSERÇÃO DA } \\
\text { ESPIGA }(\mathrm{cm})\end{array}$ & TOMBAMENTO (\%) & $\begin{array}{l}\text { PROLIFICIDADE } \\
\text { (espigas/planta) }\end{array}$ & $\begin{array}{l}\text { RENDIMENTO GRÃOS } \\
\mathrm{kg} / \mathrm{ha}(13 \%)\end{array}$ \\
\hline Local (L) & 1 & $10034,08 *$ & $99,75^{*}$ & $0,23 *$ & $126657716^{*}$ \\
\hline Repetições/ambientes & 8 & 140,50 & 4,40 & 0,02 & 7449650 \\
\hline Tratamentos & 42 & $526,62 *$ & 2,77 & $0,05^{*}$ & $6126974 *$ \\
\hline Híbridos $(\mathrm{H})$ & 35 & $474,34 *$ & 1,24 & $0,05^{*}$ & $4433993 *$ \\
\hline C.G.C. & 8 & $1337,95 *$ & 2,73 & $0,09 *$ & $9671040 *$ \\
\hline C.E.C. & 27 & $206,06^{*}$ & 0,79 & 0,04 & $2882276^{*}$ \\
\hline Testemunhas (T) & 6 & $381,29 *$ & 5,15 & 0,03 & $9204196^{*}$ \\
\hline H x.T & 1 & $32228,40 *$ & $42,04 *$ & $0,17 *$ & $46917977 *$ \\
\hline Tratamentos $\mathrm{x}$ Locais & 42 & $136,04 *$ & $1,35^{*}$ & $0,02 *$ & $1299556^{*}$ \\
\hline Híbridos x L & 35 & $139,27 *$ & $1,07 *$ & $0,02 *$ & $124679 *$ \\
\hline C.G.C. x L & 8 & $323,25^{*}$ & $1,94 *$ & $0,03 *$ & $2168447 *$ \\
\hline C.E.C. $x \mathrm{~L}$ & 27 & $85,02 *$ & 0,81 & $0,02 *$ & $996897 *$ \\
\hline Testemunhas x L & 6 & $72,35^{*}$ & $2,55 *$ & 0,01 & $1709783^{*}$ \\
\hline$(\mathrm{H} v \mathrm{~T} T) \times \mathrm{L}$ & 1 & $405,13 *$ & $3,95 *$ & $0,08 *$ & 58889 \\
\hline Erro & 206 & 30,45 & 0,67 & 0,01 & 552178 \\
\hline C.V. (\%) & & 6,00 & 45,00 & 10,60 & 8,8 \\
\hline
\end{tabular}

* Significativo pelo teste $\mathrm{F}$ em nível de $5 \%$ de probabilidade de erro.

para os tratamentos, onde o teste $\mathrm{F}$ foi significativo para os caracteres altura de inserção da espiga, prolificidade e rendimento de grãos $(\mathrm{P}<0,01)$.

Os quadrados médios para os tratamentos $\mathrm{X}$ locais foram significativos para os caracteres altura de inserção da espiga, rendimento de grãos $(\mathrm{P}<$ $0,01)$, porcentagem de tombamento e prolificidade $(\mathrm{P}<0,05)$ (Tabela 1). Os quadrados médios da C.G.C. $\mathrm{x}$ locais foram significativos para altura de inserção da espiga, porcentagem de tombamento, rendimento de grãos e prolificidade. Para C.E.C. x locais, foram observadas diferenças significativas para altura de inserção da espiga e rendimento de grãos $(\mathrm{P}<0,01)$ e, para prolificidade $(\mathrm{P}<0,05)$. A significância da interação da C.G.C. e C.E.C. com locais comprovou a diferença de comportamento dos genótipos nos dois locais. A interação da C.G.C. com local apresentou quadrados médios maiores para as características observadas, demonstrando que os efeitos aditivos foram os maiores responsáveis pelas diferenças de comportamento dos genótipos (Tabela 1). Resultados semelhantes foram obtidos por BECK et al. (1990) avaliando populações em cinco locais, e por NEVADO \& CROSS (1990), e para cruzamentos entre linhagens por MELCHINGER et al. (1990), segundo os quais os efeitos aditivos também foram os maiores responsáveis pelas variações nas médias de rendimento e nos demais caracteres avaliados. Os efeitos da C.G.C. por local e para todos os caracteres estão na tabela 2. Para altura de inserção da espiga ocorreram grandes diferenças na C.G.C. das linhagens dependendo do local. Em Não Me Toque as linhagens que contribuíram para maior redução no caráter foram IF02 e ID01; já em Tapera foram IMD05 e IMD06. Para a porcentagem de tombamento e prolificidade, não ocorreram grandes diferenças no comportamento das linhagens. Para o rendimento de grãos as melhores linhagens foram as mesmas nos dois locais.

Na tabela 3, estão apresentados os valores observados para altura de inserção da espiga superior. Os cruzamentos atingiram, em média, valores maiores em Não Me Toque $(101 \mathrm{~cm})$ do que em Tapera $(89 \mathrm{~cm})$. Isto pode explicar a porcentagem de tombamento bem menor em Tapera, mesmo com somente duas linhagens apresentando diferenças quanto às estimativas da C.G.C. nos dois locais (Tabela 2). Os cruzamentos que revelaram os maiores efeitos específicos de decréscimo para a altura de inserção da espiga superior em Não Me Toque foram IMD05 x IF08 e IF03 x ID07, e em Tapera foram ID01 x IMD06, IMD04 x ID09 e IMD05 x IF08 (Tabela 3).

A prolificidade média foi de 1,01 espigas por planta em Não Me Toque, variando de 0,91 (IMD06 x IF08) à 1,31 (IMD04 x IMD06) espigas por planta. Em Tapera, a prolificidade média foi de 1,09 espigas por planta, variando entre 0,98 (IF02 x ID09) e 1,72 espigas por planta (IF03 x IF08), conforme mostra a tabela 4. Pelas estimativas dos efeitos aditivos, somente três linhagens levaram ao 
Tabela 2 - Estimativas dos efeitos da capacidade geral de combinação ( $\left.g_{i}\right)$ dos F1's para os quatro caracteres estudados. Não Me Toque e Tapera/RS, 1992.

\begin{tabular}{|c|c|c|c|c|c|c|c|c|}
\hline \multirow{3}{*}{ LINHAGENS } & \multicolumn{6}{|c|}{ EFEITOS DA CAPACIDADE GERAL DE COMBINAÇÃO } & & \\
\hline & \multicolumn{2}{|c|}{$\begin{array}{l}\text { ALTURA INSERÇÃO } \\
\text { ESPIGA }(\mathrm{cm})\end{array}$} & \multicolumn{2}{|c|}{ TOMBAMENTO $(\%)$} & \multicolumn{2}{|c|}{$\begin{array}{l}\text { PROLIFICIDADE (espi- } \\
\text { gas/planta) }\end{array}$} & \multicolumn{2}{|c|}{$\begin{array}{c}\text { RENDIMENTO GRÃOS } \\
\text { kg/há }(13 \%)\end{array}$} \\
\hline & NMT & TAP & NMT & TAP & NMT & TAP & NMT & TAP \\
\hline ID01 & $-9,32$ & $-5,31 *$ & -024 & 0,002 & $-0,004$ & 0,005 & 978 & 788 \\
\hline IF02 & $-10,17$ & $-0,74 *$ & $-0,32$ & 0,05 & $-0,038$ & 0,117 & -641 & $232 *$ \\
\hline IF03 & $-3,32$ & $2,68^{*}$ & $-0,17$ & $-0,15$ & 0,505 & $-0,039$ & -755 & -493 \\
\hline IMD04 & 6,96 & 8,96 & $-0,29$ & $-0,11$ & 0,053 & $-0,048$ & 933 & $439 *$ \\
\hline IMD05 & $-0,32$ & $-6,60 *$ & $-0,65$ & $-0,05^{*}$ & $-0,026$ & $-0,019$ & -521 & $-1092 *$ \\
\hline IMD06 & $-1,75$ & $-5,88 *$ & 0,16 & 0,18 & $-0,004$ & $-0,066$ & -76 & 254 \\
\hline ID07 & 1,96 & $-1,88 *$ & 0,51 & $-0,009$ & $-0,038$ & 0,119 & -179 & -306 \\
\hline IF08 & 5,54 & 4,11 & 0,17 & 0,07 & $-0,0009$ & 0,119 & -287 & -169 \\
\hline ID09 & 10,39 & $4,68^{*}$ & 0,85 & $0,03 *$ & 0,007 & $-0,025$ & 548 & 347 \\
\hline d.p. $\left(g_{i}-g_{j}\right)$ & 1,61 & 1,04 & 0,35 & 0,15 & 0,047 & 1,069 & 231 & 205 \\
\hline
\end{tabular}

* Significativo pelo teste t em nível de 5\% de probabilidade de erro entre locais. NMT $=$ NÃO ME TOQUE

$\mathrm{TAP}=\mathrm{TAPERA}$

aumento do número de espigas por planta em Não Me Toque (IF03, IMD04 e ID09), tendo IF03 o maior efeito (Tabela 2). Em Tapera, três linhagens proporcionaram aumento na prolificidade (ID01,
IF03 e IF08), sendo o maior efeito apresentado por IF08. O fato de não ocorrer diferença entre as estimativas da C.G.C. nos dois locais indica que a seleção para este caráter pode ser realizada em qualquer dos ambientes.

O potencial de

Tabela 3 - Médias de altura de inserção da espiga superior $(\mathrm{cm})$ - acima da diagonal, e estimativas dos efeitos da capacidade específica de combinação $\left(\mathrm{s}_{\mathrm{ij}}\right)$ dos F1's - abaixo da diagonal, desviospadrão entre dois F1's com e sem genitor comum, em Não Me Toque e Tapera/RS, 1992.

\begin{tabular}{|c|c|c|c|c|c|c|c|c|c|c|}
\hline \multicolumn{11}{|c|}{ LINHAGENS } \\
\hline & & ID01 & IF02 & IF03 & IMD04 & IMD05 & IMD06 & ID07 & IF08 & ID09 \\
\hline ID01 & $\begin{array}{l}\text { NMT } \\
\text { TAP }\end{array}$ & & $\begin{array}{l}87 \\
89\end{array}$ & $\begin{array}{l}88 \\
91\end{array}$ & $\begin{array}{l}106 \\
93\end{array}$ & $\begin{array}{l}92 \\
68\end{array}$ & $\begin{array}{l}87 \\
63\end{array}$ & $\begin{array}{l}93 \\
91\end{array}$ & $\begin{array}{l}96 \\
92\end{array}$ & $\begin{array}{l}92 \\
88\end{array}$ \\
\hline IF02 & $\begin{array}{l}\text { NMT } \\
\text { TAP }\end{array}$ & $\begin{array}{l}5,71 \\
6,03\end{array}$ & & $\begin{array}{l}96 \\
88\end{array}$ & $\begin{array}{l}94 \\
91\end{array}$ & $\begin{array}{l}87 \\
86\end{array}$ & $\begin{array}{l}87 \\
82\end{array}$ & $\begin{array}{l}87 \\
89\end{array}$ & $\begin{array}{l}92 \\
90\end{array}$ & $\begin{array}{l}105 \\
92\end{array}$ \\
\hline IF03 & $\begin{array}{l}\text { NMT } \\
\text { TAP }\end{array}$ & $\begin{array}{l}-014 \\
4,60\end{array}$ & $\begin{array}{l}8,71 \\
-2,96\end{array}$ & & $\begin{array}{l}104 \\
100\end{array}$ & $\begin{array}{l}96 \\
83\end{array}$ & $\begin{array}{l}96 \\
87\end{array}$ & $\begin{array}{l}89 \\
84\end{array}$ & $\begin{array}{l}106 \\
90\end{array}$ & $\begin{array}{l}108 \\
108\end{array}$ \\
\hline IMD04 & $\begin{array}{l}\text { NMT } \\
\text { TAP }\end{array}$ & $\begin{array}{l}7,57 \\
0,32\end{array}$ & $\begin{array}{l}-3,57 \\
-6,25\end{array}$ & $\begin{array}{l}-0,43 \\
-0,67\end{array}$ & & $\begin{array}{l}102 \\
97\end{array}$ & $\begin{array}{l}104 \\
101\end{array}$ & $\begin{array}{l}116 \\
92\end{array}$ & $\begin{array}{l}115 \\
111\end{array}$ & $\begin{array}{l}114 \\
90\end{array}$ \\
\hline IMD05 & $\begin{array}{l}\text { NMT } \\
\text { TAP }\end{array}$ & $\begin{array}{l}0,86 \\
-9,11\end{array}$ & $\begin{array}{l}-3,28 \\
4,32\end{array}$ & $\begin{array}{l}-1,14 \\
-2,11\end{array}$ & $\begin{array}{l}-5,42 \\
5,61\end{array}$ & & $\begin{array}{l}107 \\
83\end{array}$ & $\begin{array}{l}109 \\
82\end{array}$ & $\begin{array}{l}89 \\
74\end{array}$ & $\begin{array}{l}122 \\
93\end{array}$ \\
\hline IMD06 & $\begin{array}{l}\text { NMT } \\
\text { TAP }\end{array}$ & $\begin{array}{l}-2,71 \\
-14,82\end{array}$ & $\begin{array}{l}-1,86 \\
-0,39\end{array}$ & $\begin{array}{l}0,28 \\
1,17\end{array}$ & $\begin{array}{l}-2,00 \\
8,89\end{array}$ & $\begin{array}{l}8,28 \\
6,46\end{array}$ & & $\begin{array}{l}97 \\
78\end{array}$ & $\begin{array}{l}112 \\
92\end{array}$ & $\begin{array}{l}104 \\
85\end{array}$ \\
\hline ID07 & $\begin{array}{l}\text { NMT } \\
\text { TAP }\end{array}$ & $\begin{array}{l}-0,43 \\
9,17\end{array}$ & $\begin{array}{l}-5,57 \\
2,61\end{array}$ & $\begin{array}{l}-10,43 \\
-5,82\end{array}$ & $\begin{array}{l}6,28 \\
-4,11\end{array}$ & $\begin{array}{l}6,57 \\
1,46\end{array}$ & $\begin{array}{l}-4,00 \\
-3,25\end{array}$ & & $\begin{array}{l}115 \\
93\end{array}$ & $\begin{array}{l}114 \\
90\end{array}$ \\
\hline IF08 & $\begin{array}{l}\text { NMT } \\
\text { TAP }\end{array}$ & $\begin{array}{l}-1,00 \\
4,17\end{array}$ & $\begin{array}{l}-4,14 \\
-2,39\end{array}$ & $\begin{array}{l}3,00 \\
-5,82\end{array}$ & $\begin{array}{l}1,71 \\
8,89\end{array}$ & $\begin{array}{l}-17,00 \\
-12,53\end{array}$ & $\begin{array}{l}7,43 \\
4,75\end{array}$ & $\begin{array}{l}6,71 \\
1,75\end{array}$ & & $\begin{array}{l}120 \\
99\end{array}$ \\
\hline ID09 & $\begin{array}{l}\text { NMT } \\
\text { TAP }\end{array}$ & $\begin{array}{l}-9,85 \\
-0,39\end{array}$ & $\begin{array}{l}4,00 \\
-0,96\end{array}$ & $\begin{array}{l}0,14 \\
11,61\end{array}$ & $\begin{array}{l}-4,14 \\
-12,67\end{array}$ & $\begin{array}{l}11,14 \\
5,89\end{array}$ & $\begin{array}{l}-5,43 \\
-2,82\end{array}$ & $\begin{array}{l}0,86 \\
-1,82\end{array}$ & $\begin{array}{l}3,28 \\
1,18\end{array}$ & \\
\hline
\end{tabular}

\begin{tabular}{lc}
\multicolumn{1}{c}{ NMT } & TAP \\
d.p. $\left(\mathrm{s}_{\mathrm{ij}}-\mathrm{s}_{\mathrm{ik}}\right)=3,9535$ & 2,5382 \\
d.p. $\left(\mathrm{s}_{\mathrm{ij}}-\mathrm{s}_{\mathrm{k}}\right)=3,6091$ & 2,3170 \\
NMT = NÃO ME TOQUE &
\end{tabular}

$\mathrm{NMT}=\mathrm{NÃO}$ ME TOQUE

$\mathrm{TAP}=\mathrm{TAPERA}$ rendimento de grãos atingido em Tapera foi maior do que em Não Me Toque, sendo que, devido à interação da C.E.C. com local, os híbridos mais produtivos num local não o foram no outro. O rendimento médio geral dos 36 cruzamentos foi de $8349 \mathrm{~kg} / \mathrm{ha}$ em Não Me Toque e $9712 \mathrm{~kg} / \mathrm{ha} \mathrm{em}$ Tapera. Os híbridos ID01 x IMD04 e ID01 x IMD06 obtiveram os maiores rendimentos de grãos em Não Me Toque e os híbridos ID01 x ID09 e IMD04 $\mathrm{x}$ IMD06 obtiveram os maiores rendimentos de grãos em Tapera (Tabela 5). Tanto em Não Me Toque como em Tapera, os maiores efeitos de aumento no rendimento foram das linhagens ID01 e IMD04 (Tabela 2). Apesar da 
Tabela 4 - Médias de prolificidade dos F1's de milho em Não Me Toque e Tapera/RS, 1992.

\begin{tabular}{|c|c|c|c|c|c|c|c|c|c|c|}
\hline & & ID01 & IF02 & IF03 & IMD04 & IMD05 & IMD06 & ID07 & IF08 & ID09 \\
\hline \multirow[t]{2}{*}{ ID01 } & NMT & & 0,99 & 0,97 & 1,18 & 0,98 & 1,01 & 0,99 & 0,97 & 1,05 \\
\hline & TAP & & 1,33 & 1,08 & 1,19 & 1,03 & 1,03 & 1,05 & 1,04 & 1,02 \\
\hline \multirow[t]{2}{*}{$\mathrm{IF} 02$} & NMT & & & 0,99 & 0,99 & 1,01 & 0,97 & 0,95 & 0,98 & 0,98 \\
\hline & TAP & & & 1,05 & 1,01 & 1,02 & 1,00 & 1,01 & 1,10 & 0,98 \\
\hline \multirow[t]{2}{*}{ IF03 } & NMT & & & & 1,05 & 1,04 & 1,04 & 1,01 & 1,25 & 1,17 \\
\hline & TAP & & & & 1,10 & 1,19 & 1,19 & 1,01 & 1,72 & 1,25 \\
\hline \multirow{2}{*}{ IMD04 } & NMT & & & & & 0,98 & 1,31 & 1,01 & 1,01 & 1,01 \\
\hline & TAP & & & & & 1,02 & 1,09 & 1,02 & 1,07 & 0,99 \\
\hline \multirow[t]{2}{*}{ IMD05 } & NMT & & & & & & 0,94 & 0,99 & 0,99 & 1,05 \\
\hline & TAP & & & & & & 1,04 & 1,00 & 1,12 & 1,01 \\
\hline \multirow[t]{2}{*}{ IMD06 } & NMT & & & & & & & 0,96 & 0,91 & 1,00 \\
\hline & TAP & & & & & & & 1,03 & 1,19 & 1,06 \\
\hline \multirow[t]{2}{*}{ ID07 } & NMT & & & & & & & & 1,02 & 0,97 \\
\hline & TAP & & & & & & & & 1,13 & 1,05 \\
\hline \multirow[t]{2}{*}{ IF08 } & NMT & & & & & & & & & 0,99 \\
\hline & TAP & & & & & & & & & 1,23 \\
\hline
\end{tabular}

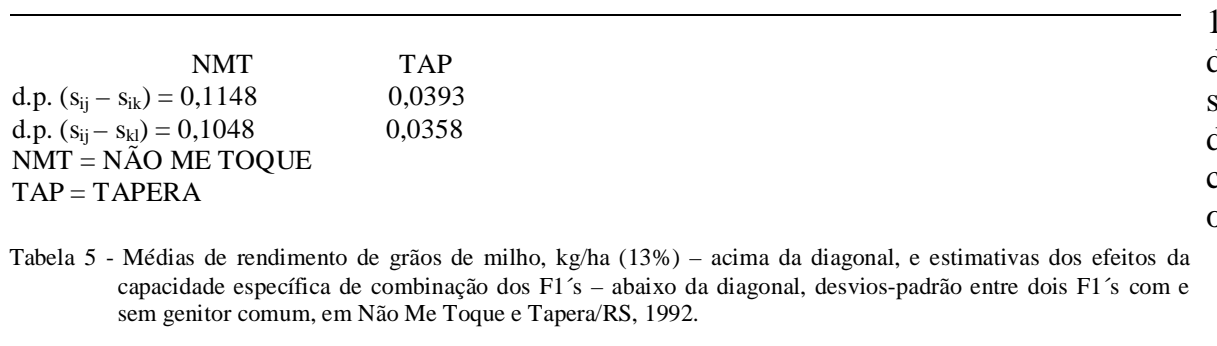

\begin{tabular}{lllllllllll}
\hline \multirow{7}{*}{} & & \multicolumn{7}{c}{ LINHAGENS } \\
\hline \multirow{2}{*}{ ID01 } & ID01 & IF02 & IF03 & IMD04 & IMD05 & IMD06 & ID07 & IF08 & ID09 \\
\cline { 3 - 10 } & NMT & & 8338 & 8162 & 10703 & 8858 & 10226 & 8338 & 9435 & 9580 \\
& TAP & & 11068 & 9618 & 10082 & 8825 & 10024 & 10070 & 11402 & 12125 \\
IF02 & NMT & $-3,47$ & & 6931 & 8782 & 7536 & 6795 & 7307 & 8420 & 8187 \\
& TAP & 335 & & 9233 & 10538 & 9803 & 9042 & 10364 & 9667 & 9605 \\
IF03 & NMT & -409 & -20 & & 8796 & 7218 & 8074 & 6276 & 7495 & 8547 \\
& TAP & -388 & -217 & & 9990 & 7998 & 9693 & 8066 & 8671 & 10967 \\
IMD04 & NMT & 440 & 140 & 268 & & 8422 & 9156 & 9245 & 8868 & 9360 \\
& TAP & -857 & 154 & 332 & & 9108 & 11938 & 9982 & 10290 & 8843 \\
IMD0 & NMT & 52 & 350 & 146 & -339 & & 6753 & 9169 & 6088 & 9093 \\
& TAP & -582 & 951 & -127 & 49 & & 9337 & 9174 & 6715 & 9086 \\
IMD06 & NMT & 974 & -835 & 557 & -51 & -997 & & 8384 & 7739 & 9127 \\
& TAP & -730 & -1106 & -220 & 1532 & 463 & & 8700 & 10557 & 10183 \\
ID07 & NMT & -809 & -219 & -1137 & 141 & 1521 & 291 & & 8405 & 8405 \\
& TAP & -123 & 726 & -845 & 137 & 861 & -959 & & 9541 & 9652 \\
IF08 & NMT & 394 & 1000 & 187 & -128 & -1451 & -246 & 523 & & 8328 \\
& TAP & 1071 & -107 & -377 & 308 & -1734 & 760 & 305 & & 9663 \\
ID09 & NMT & -295 & -68 & 405 & -471 & 717 & 306 & -312 & -281 & \\
& TAP & 1277 & -686 & 1402 & -1655 & 119 & -130 & -100 & -226 &
\end{tabular}

\begin{tabular}{ll}
\multicolumn{1}{c}{ NMT } & TAP \\
d.p. $\left(\mathrm{s}_{\mathrm{ij}}-\mathrm{s}_{\mathrm{ik}}\right)=565$ & 502 \\
d.p. $\left(\mathrm{s}_{\mathrm{ij}}-\mathrm{s}_{\mathrm{k}}\right)=516$ & 458 \\
NMT $=$ NÂO ME TOQUE &
\end{tabular}

NMT $=$ NÃO ME TOQUE

$\mathrm{TAP}=\mathrm{TAPERA}$

interação da C.G.C. com local, as linhagens presentes nas melhores combinações foram as mesmas nos dois ambientes, sendo elas ID01, IMD04, IMD06 e ID09. Estes resultados mostram que, para identificação das linhagens com melhor C.G.C., bastaria a avaliação em um dos ambientes. A interação significativa da C.E.C. com local revelou que os melhores cruzamentos para rendimento de grãos não foram os mesmos nos dois locais. Tal fato indica genótipos com adaptação específica para cada ambiente e confirma a necessidade de testes em vários locais para seleção para rendimento, conforme já relatado por outros autores (PANDEY et al., 1991). Assim, a predição de híbridos não poderia ser feita baseada em dados de um só ambiente. O cruzamento ID x IMD04 obteve o maior rendimento de grãos em Não Me Toque e um desempenho em torno da média em Tapera, indicando que uma parcela significativa dos efeitos não-aditivos foi representada pela interação do genótipo com o ambiente.

Para uma comparação com os híbridos duplos e triplos existentes no mercado do Rio Grande do Sul, foram incluídas sete testemunhas comerciais nos experimentos. $\mathrm{Na}$ tabela 6, estão os rendimentos de grãos destas testemunhas e de alguns cruzamentos que obtiveram um bom desempenho nos ensaios em Não Me Toque e Tapera. Em Não Me Toque, a média de rendimento de grãos foi de $8571 \mathrm{~kg} / \mathrm{ha}$. Em Tapera, a média de rendimento de grãos foi de $9954 \mathrm{~kg} / \mathrm{ha}$. Entre as linhagens, ID01 obteve destaque por participar de diversos cruzamentos que apresentaram rendimentos 
superiores à média. Os híbridos que poderiam ser considerados para a utilização comercial imediata seriam: ID01/IMD04 e ID01/IMD06 em Não Me Toque e ID01/ID09 e IMD04/ID09 em Tapera.

\section{CONCLUSÕES}

Quatro linhagens apresentaram características desejávais para serem empregadas no programa de melhoramento para a síntese de híbridos e na reciclagem de linhagens: ID01, IMD04, IMD06 e ID09.

Apesar das estimativas da C.G.C. e da CEC revelarem diferenças dependendo do local de teste, a CGC foi um bom indicador do desempenho das linhagens em combinações híbridas nos dois ambientes.

\section{REFERÊNCIAS BIBLIOGRÁFICAS}

BECK, D.L., VASAL, S.K., CROSSA, J. Heterosis and combining ability of CIMMYT's tropical early and intermediate maturity maize (Zea mays L.) germoplasm. Maydica, Bergamo, v.35, p.279-285, 1990.

GRIFFING, B. Concept of general and specific combining ability in relation to diallel crossing sistems. Austr J Bio Sci, East Melbourne, v.9, n.4, p.463-493, 1956

MELCHINGER, A .E., LEE, M., LAMKEY, K.R., et al. Genetic diversity for restriction fragment lenght polymorphisms and heterosis for two diallel sets of maize inbreds. Theor Appl Genetics, v.80, p.488-496, 1990.

MUNGOMA, C., POLLAK, L. M. Heterotic patterns among tem corn belt and exotic maize populations. Crop Sci, Madison, v.28, p.500-504, 1988 .

NEVADO, M.E., CROSS, H.Z. Diallel analysis of relative growth rates in maize synthetics. Crop Sci, Madison, v.30, p.549-552, 1990
Tabela 6 - Médias de rendimento de grãos de milho, kg/ha (13\%) dos melhores híbridos F1's e testemunhas comerciais, em Não Me Toque e Tape$\mathrm{ra} / \mathrm{RS}, 1992$.

\begin{tabular}{|c|c|c|c|c|c|}
\hline \multicolumn{3}{|c|}{ NÃO ME TOQUE } & \multicolumn{3}{|c|}{ TAPERA } \\
\hline GENÓTIPO & \multicolumn{2}{|c|}{$\begin{array}{l}\text { RENDIMENTO } \\
\mathrm{kg} / \text { há }(13 \%)\end{array}$} & gENÓTIPO & \multicolumn{2}{|c|}{$\begin{array}{l}\text { RENDIMENTO } \\
\mathrm{kg} / \text { há }(13 \%)\end{array}$} \\
\hline $\mathrm{T} 2$ (HD) & $11082 \mathrm{a}$ & & ID01 x ID09 & $12125 \mathrm{a}$ & \\
\hline ID01 x IMD04 & $10703 a$ & & IMD04 x IMD06 & $11938 \mathrm{a}$ & \\
\hline T6 (HTM) & 10343 & $\mathrm{~b}$ & $\mathrm{~T} 2$ & 11457 & $\mathrm{bc}$ \\
\hline ID01 x IMD06 & 10226 & $\mathrm{bc}$ & ID01 x IF08 & 11402 & bcd \\
\hline ID01 x ID09 & 9580 & $\mathrm{~cd}$ & ID01 x IF02 & 11068 & cde \\
\hline ID01 x IF08 & 9435 & de & $\mathrm{T} 7$ & 11062 & cde \\
\hline IMD04 x ID09 & 9360 & de & IF03 x ID09 & 10967 & cdef \\
\hline IMD04 x ID07 & 9245 & de & T6 & 10847 & cdefg \\
\hline IMD05 x ID07 & 9169 & de & IMD06 x IF08 & 10557 & efgh \\
\hline IMD04 x IMD06 & 9156 & de & IF02 x IMD04 & 10538 & efgh \\
\hline IMD06 x ID09 & 9127 & de & T5 & 10418 & fgh \\
\hline IMD05 x ID09 & 9093 & def & $\mathrm{T} 3$ & 10399 & fgh \\
\hline $\mathrm{T} 4$ (HD) & 8975 & def & IF02 x ID07 & 10364 & gh \\
\hline IMD04 x IF08 & 8868 & ef & IMD04 x IF08 & 10290 & $\mathrm{~h}$ \\
\hline $\mathrm{T} 7$ (HD) & 8859 & ef & IMD06 x ID09 & 10183 & $\mathrm{~h}$ \\
\hline T3 (HD) & 8576 & $\mathrm{f}$ & ID01 x IMD04 & 10082 & $\mathrm{~h}$ \\
\hline $\mathrm{T} 1$ (HD) & 8545 & $\mathrm{f}$ & $\mathrm{T} 4$ & 10033 & $\mathrm{~h}$ \\
\hline T5 (HD) & 7826 & $\mathrm{~g}$ & $\mathrm{~T} 1$ & 9419 & i \\
\hline MÉDIA & 8571 & & MÉDIA & 9954 & \\
\hline C.V. (\%) & 8,5 & & C.V. $(\%)$ & 9,4 & \\
\hline
\end{tabular}

* Médias seguidas de letras diferentes diferem significativamente pelo teste de Tukey em nível de $5 \%$ de probabilidade de erro.

PANDEY, S., VASAL, S.K., DEUTSCH, J.A. Performance of openpollinated maize cultivars selected from 10 tropical maize populations. Crop Sci, Madison, v.31, p.285-290, 1991.

PIMENTEL GOMES, F. Curso de estatística experimental. 13 ed. Piracicaba : Nobel, 1990. 468p.

VENCOVSKY, R. Herança quantitativa. In: PATERNIANI, E., VIÉGAS, G.P. (ed). Melhoramento e produção do milho. 2.ed. Campinas : Fundação Cargill, 1987. p.137-214.

VENCOVSKY, R., BARRIGA, P. Genética biométrica no fitomelhoramento. Ribeirão Preto : Soc. Bras. de Genética, 1992. 486p. 